\title{
Response: Commentary: A Reassessment of the Taxonomic Position of Mesosaurs, and a Surprising Phylogeny of Early Amniotes
}

\author{
Michel Laurin ${ }^{1 *}$ and Graciela Piñeiro ${ }^{2}$ \\ ${ }^{1}$ CR2P (UMR 7207), CNRS/MNHN Sorbonne Université, "Centre de Recherches sur la Paléobiodiversité et les \\ Paléoenvironnements", Muséum National d'Histoire Naturelle, Paris, France, ${ }^{2}$ Departamento de Paleontología, Facultad de \\ Ciencias, Montevideo, Uruguay
}

Keywords: Mesosauridae, Parareptilia, Synapsida, Sauropsida, Amniota, Paleozoic, temporal fenestration

OPEN ACCESS

Edited by:

Corwin Sullivan,

University of Alberta, Canada

Reviewed by:

Tiago Simoes,

University of Alberta, Canada

*Correspondence:

Michel Laurin

michel.laurin@mnhn.fr

Specialty section:

This article was submitted to

Paleontology,

a section of the journal

Frontiers in Earth Science

Received: 26 September 2018 Accepted: 14 November 2018 Published: 03 December 2018

Citation:

Laurin M and Piñeiro G (2018)

Response: Commentary: A

Reassessment of the Taxonomic

Position of Mesosaurs, and a

Surprising Phylogeny of Early

Amniotes. Front. Earth Sci. 6:220.

doi: 10.3389/feart.2018.00220

\section{A Commentary on}

Commentary: A Reassessment of the Taxonomic Position of Mesosaurs, and a Surprising Phylogeny of Early Amniotes

by MacDougall, M. J., Modesto, S. P., Brocklehurst, N., Verrière, A., Reisz, R. R., and Fröbisch, J. (2018). Front. Earth Sci. 6:99. doi: 10.3389/feart.2018.00099

\section{INTRODUCTION}

Mesosaurs, known from the Early Permian of southern Africa, Brazil, and Uruguay, are the oldest known amniotes with a primarily, though probably not strictly, aquatic lifestyle (Nuñez Demarco et al., 2018). Despite having attracted the attention of several prominent scientists, such as Wegener (1966), who used them to support his theory of continental drift, and the great anatomist and paleontologist von Huene (1941), who first suggested the presence of a lower temporal fenestra in Mesosaurus, several controversies still surround mesosaurs. One concerns the presence of the lower temporal fenestra in mesosaurs, which we accept (Piñeiro et al., 2012a; Laurin and Piñeiro, 2017, p. 4), contrary to Modesto $(1999,2006)$ and MacDougall et al. (2018); the other concerns the systematic position of mesosaurs, which have been argued, in the last decades, to be either the basalmost sauropsids (Laurin and Reisz, 1995; Laurin and Piñeiro, 2017), or the basalmost parareptiles (Gauthier et al., 1988; Modesto, 1999; MacDougall et al., 2018). Note that we adopt a branch-based definition of Parareptilia as Laurin and Reisz (1995) did, but use Procolophon trigoniceps as an internal specifier rather than turtles, because of the controversy surrounding the affinities about turtle origins.

In their recent response to our recent paper on the taxonomic position of mesosaurs, MacDougall et al. (2018) make a number of problematic claims, which we wish to discuss. These claims are that we used an outdated matrix and ignored over two decades of parareptile research, that our taxon selection was insufficient and that along with variability in temporal fenestration in parareptiles, all these choices explain the different taxonomic position of mesosaurs that we obtained (as the basalmost sauropsids rather than the basalmost parareptiles). Below, we respond to these claims by providing additional background data and by performing various analyses of their matrix and ours that show, through taxon and character deletion among other approaches, that neither the omission of some taxa, nor variability in temporal fenestration explains the differences 
in topologies between our study and theirs. We also highlight problems with their analyses and discuss why reusing phenotypic data matrices produced by other systematists is difficult.

\section{MATERIALS AND METHODS}

The reanalyses below use a version of our data matrix (Laurin and Piñeiro, 2017) in which we deleted characters linked to temporal fenestration, to assess the impact of this complex of characters on the resulting topology. We also reanalyze the data matrix of MacDougall et al. (2018), in three versions: unmodified, modified by ordering characters that form morphoclines, and modified further by removing the parareptile taxa not found in our matrix (Laurin and Piñeiro, 2017). We have not studied the scores of the cells of the data matrix of MacDougall et al. (2018) because this would be very time-consuming and would largely duplicate a more ambitious project focusing on early amniote phylogeny and the origin of turtles (mesosaurs are in the matrix, though the project does not focus particularly on their affinities) initiated by one of us (ML) in January 2018 in collaboration with Ingmar Werneburg, Gabriel Ferreira, and Márton Rabi.

All phylogenetic analyses were carried out with PAUP* (Swofford, 2003) version 4.0a, build 163 for Macintosh (the latest version available as of July 28,2018 ), using the branch and bound algorithm for our own data matrix, using the heuristic search with 50 random addition sequence replicates, holding 3 trees at each step, and using the tree-bisection-reconnection (TBR) algorithm for the various versions of the matrix by MacDougall et al. (2018) because that matrix had too many taxa for the branch and bound algorithm to conclude tree search in a reasonable amount of time. The branch and bound algorithm guarantees discovery of all possible most parsimonious trees. No heuristic algorithm provides a similar guarantee, but we verified for each of our analyses that all most parsimonious islands of trees had been recovered at least three times (most were recovered far more frequently and all were found at least 4 times), allowing us to be reasonably certain that we had all the most parsimonious trees. We also repeated one of these analyses (on the original version of the matrix of MacDougall et al., 2018) using 1,000 addition sequence replicates, to ensure that our settings (in particular, use of 50 addition replicates) were appropriate; we found exactly the same set of trees, which validated our choice. The results of these analyses allow us to test the main claims made by MacDougall et al. (2018).

Information associated with the analyses carried out in this paper (modified list of characters, in which we document which characters were ordered, and how the states had to be reordered to reflect the underlying continuous characters, and modified Mesquite Nexus file, which incorporates these ordering modifications) is available on the journal web site.

\section{RESULTS}

\section{Taxon Selection in Recent Studies on Mesosaur Affinities}

MacDougall et al. (2018) claim that we engage in "use of an outdated phylogenetic matrix" and that we "patently ignore over two decades of parareptilian research." These two closely related claims are factually wrong. The second point (that we ignore two decades of parareptilian research) is refuted by a simple examination of the bibliography of our paper. We did not claim to have cited all recent papers on parareptiles (given that our paper was not a review of recent studies on parareptiles), but we cited several papers published in the 1998-2018 period that discuss parareptile extensively (Reisz and Scott, 2002; Cisneros et al., 2004; Müller and Tsuji, 2007; Modesto et al., 2009; Lyson et al., 2010, 2013; Tsuji et al., 2010, 2012; Lee, 2013; Bever et al., 2015). Note that this list does not include papers about mesosaurs, which our results suggest are not parareptiles. The first point (that the matrix is obsolete) is equally factually wrong as shown by the fact that we extensively updated the original matrix (Laurin and Reisz, 1995) using both the literature and direct observations of specimens, especially mesosaurs, and explained this clearly (Laurin and Piñeiro, 2017, p. 4). For instance, Owenetta, which we added to the matrix (it was not in the matrix of Laurin and Reisz, 1995), was scored using the detailed description of Reisz and Scott (2002). We also added one of the basalmost, best-known parareptiles (Acleistorhinus), to better test previous suggestions that mesosaurs are basal parareptiles, and made other changes to the taxon set (see below). Given all these changes, we do not consider that we used an outdated phylogenetic matrix.

MacDougall et al. (2018) object to the use of suprageneric taxa as terminals because they claim that the resulting higher rate of polymorphism can weaken support values. We think that the interpretation of support values is more complex than MacDougall et al. (2018) suggest because these values tend to reflect character congruence and the number of characters (or the ratio between number of characters and number of OTUs). For many taxa, various mutually incompatible phylogenies with good support values have been published, which shows that such values should not be equated with reliability (Marjanović and Laurin, 2018). The famous case of "The guinea-pig is not a rodent" (D'Erchia et al., 1996) illustrates this point. Furthermore, while the increased polymorphism in OTUs corresponding to large clades is unavoidable and simply reflects reality, the relationship between that elevated rate of polymorphism and support values in a matrix is far more complex than suggested by MacDougall et al. (2018). This is illustrated by the fact that the taxa (typically ranked as genera) that they introduced to replace the OTU Synapsida yield a tree that suggests that this taxon, as currently delimited, is paraphyletic! Of course, a monophyletic Synapsida could still be recognized in accordance with the definition of this clade that has been proposed under the PhyloCode (using Cynognathus crateronotus as the sole internal specifier, and three extant sauropsids as external specifiers), but in their most parsimonious trees (and under both of their analyses) varanopids and the presumed ophiacodontid Archaeothyris would move out of Synapsida and become basal sauropsids (if we assume Cynognathus to be closer to edaphosaurids than to other taxa included in the tree). Thus, the support value for Synapsida in their tree (not provided) is presumably very low (bootstrap values) to negative (Bremer or decay index), depending on which index is used to assess it. Nevertheless, we moved to some degree in the direction of selecting smaller terminal taxa by breaking 
up the Testudines OTU present in Laurin and Reisz (1995) into Odontochelys, Proganochelys and Chelonii, the last of which could be broken up further in subsequent studies.

We recognize that using lower-level taxa is beneficial. The small OTUs that replace Synapsida in MacDougall et al.'s (2018) matrix yielded a new hypothesis about the position of varanopids (outside Synapsida), and this is an interesting result. As epitomized in "A hitchhiker's guide to the galaxy" (Adams, 2017), it is more important to have a good question than a precise answer, and in phylogenetics, it is perhaps more important to get interesting results that raise new questions (about the monophyly of Synapsida and Diapsida as currently delimited, for instance) than to obtain high support values which, in some cases, may simply reflect the fact that only a trivial question was asked (about affinities between a small set of closely related taxa, for instance). Note further that there is no special justification for using nominal genera as OTUs given the subjective nature of Linnaean categories; all taxonomic ranks, even species, are artificial constructs, ontologically empty designations (Ereshefsky, 2002). In addition, all taxa represent phylogenetic hypotheses, so the most rigorous approach would be to do a specimen-level analysis, though this would result in much more missing data than working on taxa, which would in turn raise new problems (e.g., Simmons, 2012a,b).

\section{Phylogeny and Evolution of Permo-Carboniferous Amniotes}

The finding by MacDougall et al. (2018) that Synapsida, as defined under the draft PhyloCode (Laurin and Reisz, in press) may exclude varanopids and the ophiacodontid Archaeothyris (other ophiacodontids were not included), which appear at the base of Sauropsida in their trees, raises two important points that MacDougall et al. (2018) did not discuss. First, it now seems likely that the phylogeny of Permo-Carboniferous amniotes is much less robust than previously thought, given that our analysis also found unorthodox results (parareptiles nested within diapsids). This is also highlighted by the fact that protorothyridids (represented by Paleothyris and Protorothyris) are paraphyletic in their consensus trees.

Second, their topology, if correct, implies that the lower temporal fenestra is an amniote synapomorphy, a possibility that we suggested earlier (Piñeiro et al., 2012a) on the basis of the presence of a fenestra in mesosaurs. MacDougall et al. (2018) clearly viewed this part of their tree as preliminary and problematic, but it is a result that should be investigated further, as they pointed out, and it adds some support to the hypothesis that the lower temporal fenestra is an amniote synapomorphy. They also discussed fenestration extensively and suggested that its great variability in amniotes both decreased its taxonomic value, a point that we already made (Piñeiro et al., 2012a), and raised doubts about our results. This last point is misleading because our matrix includes only two characters directly linked to temporal fenestration (characters 30 and 31). MacDougall et al. (2018), in their first analysis, emphasized temporal fenestration much more than we did by adding four new characters linked to temporal fenestration (their characters
171-174), even though the older version of their matrix already had three other characters also linked with temporal fenestration (their characters 44-46), in addition to their characters 42 and 43, which are the same as our characters 30 and 31 . This may have resulted in undue weight being given to temporal fenestration in analyses including all these characters. They removed all these characters linked with temporal fenestration in their second analysis to assess the impact of fenestration on their results; this impact is apparently negligible, which does not support their claim.

MacDougall et al. (2018) criticized us for accepting our own anatomical interpretations (rather than theirs) about the temporal fenestra of mesosaurs: "the authors adhere to the interpretation of Piñeiro et al. (2012a) that Mesosaurus possessed a lower lateral temporal fenestra, a condition that actually may be absent or ontogenetically variable within the taxon." This is a strange comment given that science is based on observation rather than authority, and that we have had access to far more specimens than they to support our interpretations. Should scientists prefer others' opinions over their own observations? This would run counter to the most basic scientific principles. We have seen no good evidence for absence of the fenestra in any mesosaur so far, even though many specimens are not sufficiently well-preserved to yield decisive evidence on this point.

They also claim that "specimens with supposed temporal fenestration, such as that presented in Piñeiro et al. (2012a), are extremely poorly preserved." This is misleading. Our specimens preserve bone (Figures 1C-E), whereas the specimens studied by Modesto lacked bone (Modesto, 2006, p. 347): “All specimens of Mesosaurus tenuidens examined here are preserved as natural molds in black shale. These were cast in latex rubber and drawn from photographs or by use of a camera lucida." Thus, we believe that at least some of our specimens are better-preserved than those described by Modesto, which is not surprising given that the Mangrullo Formation, from which most of our specimens originate, is a recognized Konservat-Lagerstätte (Piñeiro et al., 2012b). No author of MacDougall et al. (2018) saw more than a small proportion of the specimens that we have studied, so they are not in a good position to discuss preservation of the specimens that support our interpretation. In any case, quality of preservation can be assessed through several criteria, such as whether or not bone is present (although some external molds beautifully reconstruct the original anatomy of the bones), the degree of flattening of the skeleton, and whether or not elements are broken and disarticulated. According to three of these four criteria (presence of bone, articulation, and whether or not bones are broken), our specimens are better than those studied by Modesto (2006) because in the latter, the temporal region is disarticulated, bone is absent (only impressions remain), and several elements appear to be incompletely preserved, with broken edges, as shown by the high variability of the shape of the squamosal in the specimens illustrated by Modesto (2006). Also, disarticulation of the specimens studied by Modesto is such that in one case the basisphenoid is visible in dorsal view (Modesto, 2006, Figure 3) and in another the epipterygoid is visible in lateral view (Modesto, 2006, Figure 6). In the latter specimen, it is obvious that the jugal is bifurcated posteriorly and 

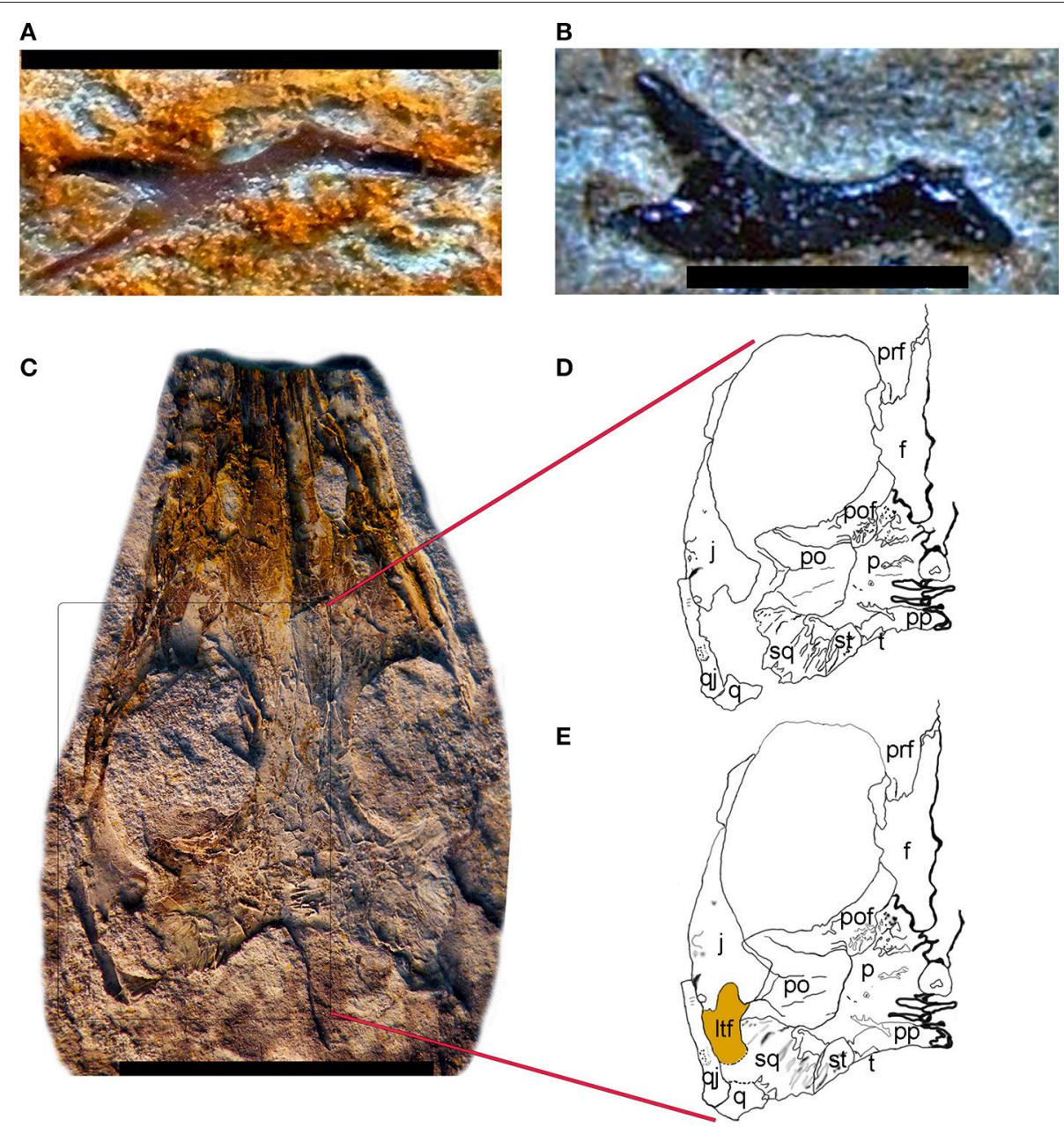

FIGURE 1 | Mesosaur lateral temporal fenestra. (A) FC-DPV 1462, right jugal, probably of a newborn individual showing the typical triradiate structure normally associated with a temporal fenestra. (B) FC-DPV 1083, right jugal of a juvenile individual showing the same typical triradiate structure. (C) FC-DPV 2534B, partial skull of an adult Mesosaurus tenuidens showing a well-preserved temporal region. Some of the bones that delimit the lateral temporal fenestra (jugal and quadratojugal) are partly disarticulated. However, the jugal displays the typical triradiate structure commonly associated with the presence of a temporal fenestra. (D) Schematic drawing of the FC-DPV 2534B left postorbital region as it was preserved, showing the jugal and quadratojugal partially disarticulated. (E) Schematic reconstruction of the possible natural anatomical configuration of the FC-DPV 2534B left postorbital region. The jugal-postorbital contact and the position of the quadratojugal relative to the jugal are approximate given that the skull bones were compressed by sediment deposition, disrupting their original three dimensional arrangement. However, the proposed configuration is the most plausible one given the morphology observed in other specimens, and the general anatomy of the bones in question. Scale: (A,B) $5 \mathrm{~mm}$; (C) $10 \mathrm{~mm}$.

defined the anteroventral corner of the lower temporal fenestra, though Modesto (2006, p. 352) interpreted this region differently. Modesto (2006) argued that the squamosal has a complementary shape and overlapped the posterior edge of the jugal, but given the extreme variability in the preserved portion of the squamosals illustrated by Modesto (2006), this interpretation seems to rest on tenuous evidence. Regarding the other preservational criterion (flattening), quality is equivalent between the specimens from Uruguay and those from Brazil and South Africa studied by Modesto $(2006 ; 2018)$. One last point to consider is that the Mangrullo formation of Uruguay has yielded isolated elements, including those that border the temporal fenestra (Figures 1A,B), and these support our interpretation.
Aside from issues of preservation of the specimens from Uruguay compared with those of Brazil and South Africa, it is clear that some specimens from Brazil display a wellpreserved temporal region featuring a lower temporal fenestra (Laurin and Piñeiro, 2017, Figure 1). Moreover, we are not the first to interpret the temporal region of Mesosaurus tenuidens specimens from the Iratí formation (Brazil) as displaying a lower temporal fenestra; our great predecessor von Huene (1941) illustrated, described, reconstructed, and discussed the systematic significance of that fenestra.

MacDougall et al. (2018) claim that "Laurin and Piñeiro made no effort to reexamine the Mesosaurus specimens that had been previously described by Modesto (2006; 2018)." This 
is again misleading at best; one of us (GP) examined many specimens from the collection studied by Modesto (though not the specimens that he illustrated) which were available as casts in Frankfurt, and has studied good photos of specimens from the American Museum of Natural History. Modesto has not studied the Brazilian collections containing thousands of mesosaur specimens; he apparently examined materials housed in South African museums and institutions, and the best specimens from the Iratí Formation in European and North American collections. Thus, one of us (GP) has examined far more mesosaur specimens (79 are mentioned in our papers; see Supplementary Data Sheet 1) than Modesto (we counted only 36 specimens mentioned in his thesis and his various papers); see Supplementary Data Sheet 1 for the source of these numbers. More importantly, why has Modesto (2006) studied only one (GPIT [Institut und Museum für Geologie und Paläontologie der Universität Tübingen] 1757-1) of the many specimens (33 are illustrated) studied by von Huene (1941), which collectively led this great paleontologist and anatomist to conclude that mesosaurs had a lower temporal fenestra? The specimen (GPIT 1757-1) studied by Modesto (2006) is exposed in dorsal view and is not very informative about the temporal region. Thus, we believe that on this front, our study rests on better grounds than that of MacDougall et al. (2018).

Nevertheless, we take this opportunity to assess the impact of temporal fenestration on our analysis by analyzing our matrix again without the two temporal fenestration characters that we originally included (our characters 30 and 31). The search, carried out in PAUP* 4.0a (build 163) for Macintosh (the latest version available as of July 28,2018 ) using the branch and bound algorithm, yielded two most parsimonious tree of 328 steps (consistency index of 0.497; homoplasy index of 0.503 ; retention index of 0.6626 ), whose strict consensus is identical with the tree that we published (Laurin and Piñeiro, 2017, Figure 5). Thus, as with MacDougall et al.'s (2018) matrix, exclusion of the temporal fenestration characters does not change the results in any significant way. This refutes the claim by MacDougall et al. (2018, p. 5) that the variability of the temporal fenestration explains the topology that we obtained.

We also reanalyzed the matrix by MacDougall et al. (2018), using the version posted on the journal's web site, initially without any modifications. We used a heuristic search with 50 random addition sequence replicates, holding 3 trees at each step, and using the tree-bisection-reconnection (TBR) algorithm. Surprisingly, we found not 9 optimal trees of 669 steps as reported by MacDougall et al. (2018), but 225 of the same length (669 steps). The strict consensus (Figure 2A; illustrated here to facilitate comparisons with our other results presented below) is identical to theirs (MacDougall et al., 2018, Figure 1A), so the mismatch in the number of most parsimonious trees might possibly attributed to subtle settings in PAUP $*$ such as whether or not zero-length branches are automatically collapsed, but we note that their tree was rooted improperly as it implied an "anamniote" clade including Seymouria and diadectomorphs that excluded amniotes, whereas there is a fairly widespread consensus that diadectomorphs are more closely related to amniotes than to seymouriamorphs (e.g., Laurin and Reisz, 1995; Ruta and Coates,
2007; Marjanović and Laurin, 2018). The rooting option of any phylogenetic tree is a hypothesis, not a result.

MacDougall et al. (2018) did not order any characters. However, simulations have shown that for characters that form morphoclines (including all characters that represent discretization of an inherently continuous variable, such as size or ratios between measurements), ordering states leads to better results, in terms of both power to recover true clades and avoidance of erroneous clades (Rineau et al., 2015, 2018). Thus, we have ordered the following multi-state characters (their numbering): 6, 47, 48, 52, 69, 89, 105, 129, 132, 147, 150, $165,167,168,178$. In some cases, we had to reorder states because the order in which they were listed made no sense if the character were to be ordered linearly, which is the simplest approach and also the one that best reflects the underlying quantitative (continuous) character. For instance, character 6, "Pineal foramen position: in the middle of the body of the parietal (0); displaced posteriorly (1); displaced anteriorly (2)" was reordered by inverting states 0 and 1 . Characters 47, 89, 147, and 178 were likewise reordered. Character 147, pertaining to humeral morphology, requires an explanation. MacDougall et al. (2018: Supplementary Data Sheet 1) indicate that this is "Modified from (Laurin and Reisz, 1995) \#104." The only modification that we see is the deletion of the ratio that served to make the state definitions more objective. In Laurin and Reisz (1995), we had indicated that the states depended on the ratio between distal humeral head width and humeral length, using 35 and $65 \%$ as thresholds. With this information, it is clear that the states should be ordered. In the list of characters (see Supplementary Data Sheet 2), we have tracked the changes made to state numbering to facilitate comparisons between our settings and those used by MacDougall et al. (2018). We also provide the revised data matrix (Supplementary Data Sheet 3) with the states reordered and ordering (or lack thereof) of multi-state characters specified, to facilitate its use by other scientists.

Analysis of the matrix with some characters ordered (as mentioned above), conducted with the same settings as previously, yielded 1,560 trees requiring 679 steps each and with a consistency index of 0.2975 , a homoplasy index of 0.7025 , and a retention index of 0.6446 . Their strict consensus (Figure 2B), unsurprisingly, is less resolved than the tree reported by MacDougall et al. (2018, Figure 1A). Notably, polytomies occur near the base of Parareptilia, in the clade that includes all parareptiles except for Milleretta, and at the base of Lanthanosuchoidea. Yet another polytomy is formed by Nyctiphruretidae, Procolophonoidea, and a clade that includes Pareiasauria and nycteroleterids. Furthermore, Nycteroleteridae may be paraphyletic as its component taxa form a polytomy that also includes Pareiasauria. This last polytomy was also obtained by MacDougall et al. (2018, Figure 1B) when they excluded the characters linked with temporal fenestration, so the monophyly of Nycteroleteridae might be worth reassessing. It might be tempting to view the lower resolution of our tree as a refutation of our ordering scheme, but it is more likely that the clades found by MacDougall et al. (2018, Figure 1A) are erroneous because simulations have shown that not ordering intrinsically 

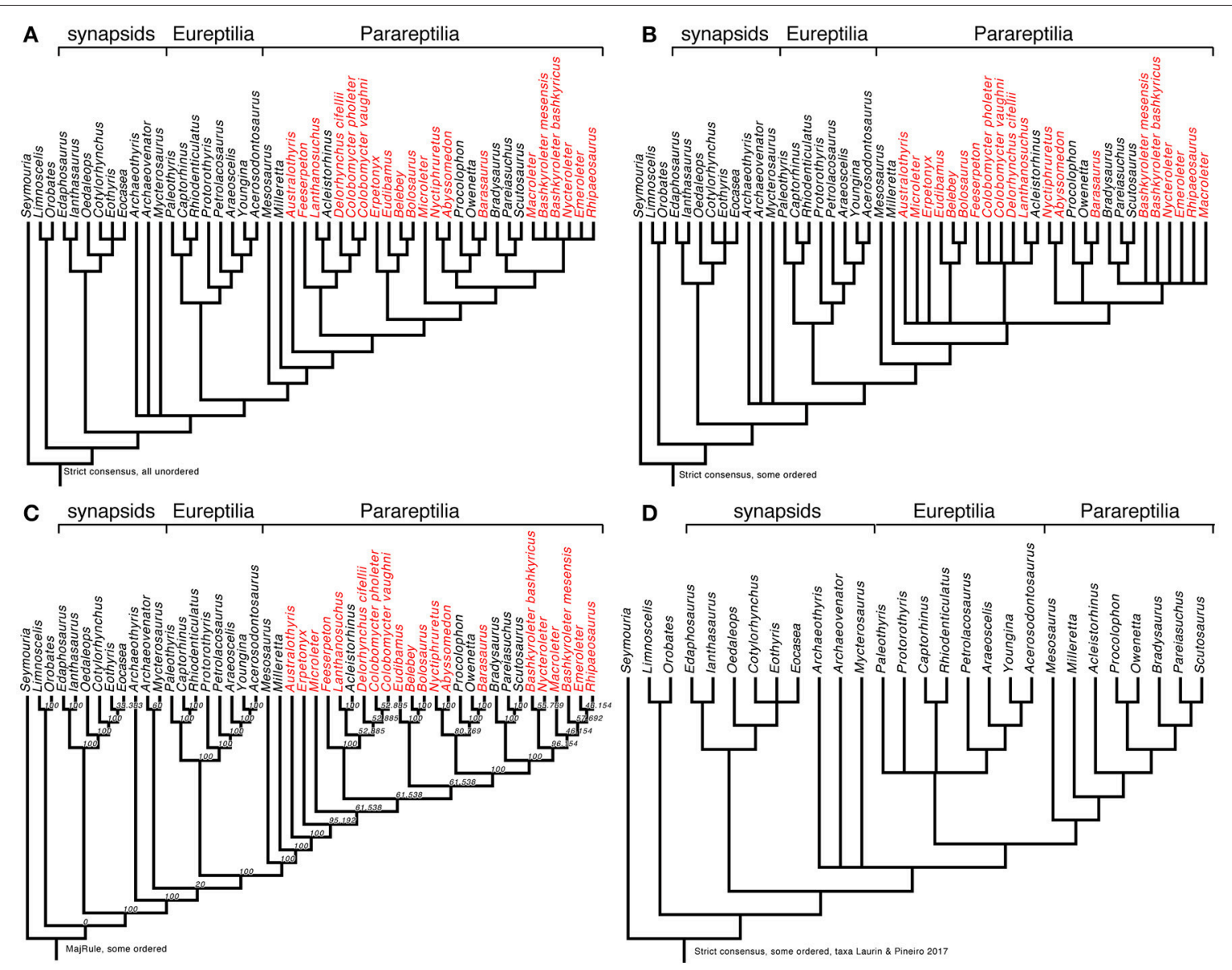

FIGURE 2 | Early amniote phylogeny as assessed through various reanalyses of a slightly modified version of the matrix of MacDougall et al. (2018). (A) Strict consensus obtained by reanalyzing the data matrix without any modifications. This is basically identical with the tree obtained by MacDougall et al. (2018, Figure $1 \mathrm{~A}$ ), but it is reproduced here to facilitate comparisons among the results obtained in our various analyses. (B) Strict consensus obtained by reanalyzing the data matrix after ordering characters $6,47,48,52,69,89,105,129,132,147,150,165,167,168,178$. Note that in some cases, the order of the states had to be altered to reflect the nature of the morphocline. (C) Majority-rule consensus tree (nodal values represent frequencies in the source trees) from the same analysis. (D) Strict consensus tree from analysis of the same matrix, except that taxa not represented in Laurin and Piñeiro (2017) were removed before analyzing the data. This refutes the claim that the topology obtained by Laurin and Piñeiro (2017) results from exclusion of several parareptile taxa (in red on parts $\mathbf{A}-\mathbf{C}$ ) that were considered by MacDougall et al. (2018), because exclusion of these taxa from the matrix of MacDougall et al. (2018) does not change the topology.

ordered characters yields a greater proportion of erroneous clades (Rineau et al., 2015, 2018).

The majority-rule consensus of the 1,560 trees obtained with some states ordered (Figure $2 \mathrm{C}$ ) reveals some interesting information not provided by MacDougall et al. (2018). Namely, varanopids may be more closely related to other sauropsids than to Archaeothyris. This result is admittedly very poorly supported (occurring in only $20 \%$ of the most parsimonious trees), but is also found (with the same frequency) when no states are ordered (not shown here, but available in Supplementary Data Sheet 3).

MacDougall et al. (2018 p. 5) claimed that the omission of several parareptile taxa in our matrix explained our topology (which placed mesosaurs outside Parareptilia). To test this hypothesis, we deleted from their matrix the parareptiles that they included but that were excluded from our matrix, although we did not remove low-ranking taxa belonging to higher-ranking taxa that we included. Thus, the OTUs belonging to Pareiasauria included in MacDougall et al. (2018) were retained, but we excluded Australothyris, Microleter, Nyctiphruretus, Barasaurus, Bashkyroleter mesensis, Bashkyroleter bashkyricus, Nycteroleter, Emeroleter, Ripaeosaurus, Lanthanosuchus, Feeserpeton, Colobomycter pholeter, Colobomycter vaughni, Delorhynchus cifellii, Abyssomedon, Eudibamus, Belebey, Erpetonyx, and Bolosaurus. The search, conducted with the same settings as above, yielded 18 trees of 465 steps, with a consistency index of 0.4151 , a homoplasy index of 0.5849 , and a retention index of 0.6397 . Their strict consensus resembles closely the tree published by MacDougall et al. (2018, Figure 1A), with Mesosaurus as the basalmost parareptile, and varanopids and the presumed ophiacodontid Archaeothyris appearing at the base of Sauropsida (Figure 2D). We also repeated this analysis with the characters linked to temporal fenestration excluded, 
as in MacDougall et al.'s (2018) second analysis. The results (not shown, but available in Supplementary Data Sheet 4) show a very similar topology, with Mesosaurus at the base of Parareptilia, varanopids, and Archaeothyris forming a polytomy at the base of Sauropsida, etc. This, along with the fact that exclusion of the temporal fenestration characters from our own matrix does not change the resulting topology, directly refutes the conclusion by MacDougall et al. (2018, p. 5) that "we illustrate that the lack of taxa in their matrix combined with the variability of temporal fenestration in Reptilia are likely contributing to the tree topology that they obtained in their phylogenetic analysis...". Obviously, the differences (including in the position of mesosaurs) in the topologies supported by our matrix and theirs must rather be attributed to discrepancies in character scoring and selection.

\section{DISCUSSION}

The differences in character treatment mentioned above (including the decision to order or not order the states, the numbering of the states, and most importantly, the way in which the states are delimited) partly explain why we did not wish to build our matrix on the basis of more recent versions of the Laurin and Reisz (1995) matrix, which were produced in Reisz's lab after one of us (ML) left that lab, or other matrices with much more tenuous links with that matrix (e.g., Schoch and Sues, 2018). In addition to the minor problems discussed above, our experience has convinced us that it is very difficult for one systematist to expand another systematist's matrix because a certain amount of subjective judgment is involved in character scoring, and each systematist has his or her own perception of a given character state. The only way to ensure coherent application of character state definitions is for a single systematist to score a given character for all taxa included in a matrix. Thus, to adequately reuse another systematist's matrix, it is unfortunately necessary to more or less redo all the scoring, after having checked, as we did, which characters should be ordered and how the ordering should be conducted. Several of these problems were among those recently discussed, along with potential solutions, by Simões et al. (2017). And of course, is it desirable to have studied specimens directly, as illustrations only provide limited information, even though recent $3 \mathrm{D}$ imaging developments have led to improvements on that front (e.g., Tissier et al., 2017).

MacDougall et al. (2018) argue that recent works have attempted to solve these various problems by improving on previous state definitions, but our own examination of recentlypublished data matrices leads us to believe that we are unfortunately still very far from that ideal situation. In fact, some of the changes introduced by MacDougall et al. (2018), such as their removal of the ratio and threshold values used to give their character 147 (humeral morphology) a quantitative basis by Laurin and Reisz (1995), make character scoring less replicable, so their work certainly does not back up their claim that substantial progress has been made on this front (at least, in the last two decades). Updating other systematists' matrices is extremely time-consuming if done carefully, as illustrated by the reanalysis of the matrix by Ruta and Coates (2007) initiated by one of us (ML) and first tackled in the doctoral thesis of Germain (2008), continued in another doctoral thesis (Marjanović, 2010), and recently published as a pre-print, in three successive versions in 2015, 2016, and 2018. This work has been under review since 2015 and will be published soon (Marjanović and Laurin, 2018). Even though this is an extreme case with respect to the time investment it required, it does not represent the most thorough rescoring of a matrix that we have performed. Rather, our work on a much smaller data matrix of 23 taxa and 42 characters, in which it was feasible to re-examine the scoring of all cells, resulted in changes in the scores assigned to $35 \%$ of the cells of the matrix, spread over all taxa and all but two characters (Marjanović and Laurin, 2008). These examples well illustrate the point that reusing other people's data matrices is not a trivial exercise, at least if it is to be done correctly, contrary to MacDougall et al.'s (2018) claim. By starting from one of our own matrices, we minimized this problem, although we did not eliminate it completely. According to McShea (2000, p. 330), "It has been said that most scientists would rather use another scientist's toothbrush than his terminology." We feel the same about other authors' phenotypic data matrices (and the stakes in this case are much higher), unless time is taken to study thoroughly each character and its distribution to ensure that whoever reworks a matrix has the same understanding about each character state as the original author. To sum up, our method did not "patently ignore over two decades of parareptilian research," and the resulting matrix is not outdated; it results from a deliberate choice to obtain a reasonably reliable matrix with an appropriate taxonomic sample for a reasonable time investment. It is a compromise, and as such, we recognize that it is imperfect and that it can be improved; we only disagree about how this should be done. Our preferred path is to work with a matrix that we know well and expand it ourselves, rather than rely on a patchwork containing additions by several scientists who may not have understood their predecessors' concept of each character.

We showed, through the new analyses presented above, that taxon selection and temporal fenestration variability do not appear to explain different topologies obtained by our group and MacDougall et al. (2018). Strangely, MacDougall et al. (2018) had all the data required to check these claims themselves but did not perform the necessary analyses before publishing their conclusions. At least one other reasonably recent phylogenetic analysis recovered mesosaurs in a fairly basal (though unresolved) position among amniotes, outside Parareptilia (Hill, 2005).

MacDougall et al. (2018) also failed to recognize that differences between our taxon selection and theirs reflect different strategies and goals. Rather than trying to sample densely parareptiles, we included three turtle OTUs (Odontochelys, Proganochelys, and Chelonii) because turtles may be parareptiles (Laurin and Reisz, 1995) and because the presence of this extant taxon in the matrix could have altered the most parsimonious topology, given their strongly divergent morphology (compared to parareptiles and diapsids). Thus, 
including turtles may be a more important way of minimizing phylogenetic artifacts than adding more parareptile taxa that strongly resemble those already present in our matrix, though the taxon deletion tests that we carried out (Laurin and Piñeiro, 2017) neither confirm nor refute this possibility. The origin of turtles remains one of the great controversies of vertebrate phylogeny (e.g., Lyson et al., 2010, 2013; Lee, 2013), and it is potentially relevant to many zoologists. Our matrix, which we will continue to develop by adding taxa and characters (through the project involving I. Werneburg, G. Ferreira, and M. Rabi evoked above), is a step toward resolving this problem. MacDougall et al. (2018) built their matrix for a more limited goal (not looking beyond Permo-Triassic taxa), and both approaches are valid and complementary. Of course, it is possible to include both many additional parareptile taxa and turtles, but the more taxa are added, the more characters need to be included to resolve their relationships. Given that research time is limited, the more cells a matrix includes, the less time goes into scoring each of them. In this respect, we agree with Simões et al. (2017) that too much attention has been given to data quantity, to the detriment of data quality, in many recent phylogenetic analyses of morphological data. Thus, there is an optimal number of taxa and characters for phenotypic matrices; more is not always better.

The fact that six authors collaborated to publish a short paper (MacDougall et al., 2018) in response to our own paper (which has only two co-authors) might be interpreted as an indication that the response paper carries the strength of consensus among a significant proportion of the (very small) community of experts on Permo-Carboniferous amniotes. However, multiple factors may have contributed to this, including the fact that these authors had collaborated in previous works, that one of them

\section{REFERENCES}

Adams, D. (2017). The Hitchhiker's Guide to the Galaxy Omnibus: A Trilogy in Five Parts. Basingstoke: Pan Macmillan.

Bever, G., Lyson, T. R., Field, D. J., and Bhullar, B.-A. S. (2015). Evolutionary origin of the turtle skull. Nature 525, 239-242. doi: 10.1038/nature14900

Cisneros, J. C., Damiani, R., Schultz, C., da Rosa, Á., Schwanke, C., Neto, L. W., et al. (2004). A procolophonoid reptile with temporal fenestration from the Middle Triassic of Brazil. Proc. R. Soc. Lond. B 271, 1541-1546. doi: $10.1098 / \mathrm{rspb} .2004 .2748$

D’Erchia, A. M., Gissi, C., Pesole, G., Saccone, C., and Arnason, U. (1996). The guinea-pig is not a rodent. Nature 381, 597-600. doi: 10.1038/381597a0

Ereshefsky, M. (2002). Linnaean ranks: Vestiges of a bygone era. Phil. Sci. 69, S305-S315. doi: 10.1086/341854

Gauthier, J., Kluge, A. G., and Rowe, T. (1988). "The early evolution of the Amniota," in The Phylogeny and Classification of the Tetrapods, Volume 1: Amphibians, Reptiles, Birds, ed M. J. Benton (Oxford: Clarendon Press),103-155.

Germain, D. (2008). Anatomie des Lépospondyles et origine des Lissamphibiens. Doctoral dissertation, Muséum National d'Histoire Naturelle, Paris, 351.

Hawking, S. (1993). A Brief History of Time. Beverly Hills, CA: Dove Audio.

Hill, R. V. (2005). Integration of morphological data sets for phylogenetic analysis of Amniota: the importance of integumentary characters and increased taxonomic sampling. Syst. Biol. 54, 530-547. doi: 10.1080/106351505909 50326

Laurin, M., and Piñeiro, G. (2017). A reassessment of the taxonomic position of mesosaurs, and a surprising phylogeny of early amniotes. Front. Earth Sci. 5:88. doi: 10.3389/feart.2017.00088 supervised the thesis of another, etc. Regardless of these possible explanations for the number of authors, we note that majority opinion has never been a safe indicator of scientific accuracy. This is illustrated by the pamphlet " 100 Authors against Einstein" (in which case the reason for the high number of authors was clear, contrary to the present situation), which attempted to refute Einstein's theory of relativity. Einstein reportedly replied (Hawking, 1993, p. 98) "If I were wrong, then one [author] would have been enough!"

\section{AUTHOR CONTRIBUTIONS}

ML planned this research, carried it out and wrote most of the draft. GP wrote part of the text, drafted Figure 1, and provided comments to improve other parts of the text.

\section{ACKNOWLEDGMENTS}

We thank Mark MacDougall for sending the draft of the paper and the supplements before their publication (but after definitive acceptance of their paper), at our request. The draft was improved by comments from David Marjanović, the handling editor Corwin Sullivan, and two other reviewers. This work was financed by a recurring grant from the French Ministry of Research and the CNRS to the CR2P (for ML) and by a grant from ANII (GP).

\section{SUPPLEMENTARY MATERIAL}

The Supplementary Material for this article can be found online at: https://www.frontiersin.org/articles/10.3389/feart. 2018.00220/full\#supplementary-material

Laurin, M., and Reisz, R. R. (1995). A reevaluation of early amniote phylogeny. Zool. J. Linn. Soc. 113, 165-223. doi: 10.1111/j.1096-3642.1995.tb00932.x

Laurin, M., and Reisz, R. R. (in press). "Synapsida," in Phylonyms: An Implementation of PhyloCode, eds P. D. Cantino, K. de Queiroz, and J. A. Gauthier (Berkeley, CA: University of California Press).

Lee, M. S. Y. (2013). Turtle origins: insights from phylogenetic retrofitting and molecular scaffolds. J. Evol. Biol. 26, 2729-2738. doi: 10.1111/jeb.12268

Lyson, T. R., Bever, G. S., Bhullar, B.-A. S., Joyce, W. G., and Gauthier, J. A. (2010). Transitional fossils and the origin of turtles. Biol. Lett. 6, 830-833. doi: 10.1098/rsbl.2010.0371

Lyson, T. R., Bever, G. S., Scheyer, T. M., Hsiang, A. Y., and Gauthier, J. A. (2013). Evolutionary origin of the turtle shell. Curr. Biol. 23, 1113-1119. doi: 10.1016/j.cub.2013.05.003

MacDougall, M. J., Modesto, S. P., Brocklehurst, N., Verrière, A., Reisz, R. R., and Fröbisch, J. (2018). Response: a reassessment of the taxonomic position of mesosaurs, and a surprising phylogeny of early Amniotes. Front. Earth Sci. 6:99. doi: 10.3389/feart.2018.00099

Marjanović, D. (2010). Phylogeny of the Limbed Vertebrates With Special Consideration of the Origin of the Modern Amphibians. U. Pierre \& Marie Curie, University of Vienna, Paris; Vienna.

Marjanović, D., and Laurin, M. (2008). A reevaluation of the evidence supporting an unorthodox hypothesis on the origin of extant amphibians. Contrib. Zool. 77, 149-199.

Marjanović, D., and Laurin, M. (2018). Phylogeny of Paleozoic limbed vertebrates reassessed through revision and expansion of the largest published relevant data matrix.PeerJ. 6:e5565. doi: 10.7717/peerj.5565

McShea, D. W. (2000). Trends, tools and terminology. Paleobiology 26, 330-333. doi: 10.1666/0094-8373(2000)026<0330:TTAT>2.0.CO;2 
Modesto, S. (2006). The cranial skeleton of the Early Permian aquatic reptile Mesosaurus tenuidens: implications for relationships and palaeobiology. Zool. J. Linn. Soc. 146, 345-368. doi: 10.1111/j.1096-3642.2006.00205.x

Modesto, S. P. (1999). Observations on the structure of the early Permian reptile Stereosternum tumidum Cope. Palaeont. Afr. 35, 7-19.

Modesto, S. P. (2010). The postcranial skeleton of the aquatic parareptile Mesosaurus tenuidens from the Gondwanan Permian. J. Vertebr. Paleontol. 30, 1378-1395. doi: 10.1080/02724634.2010.501443

Modesto, S. P., Scott, D. M., and Reisz, R. R. (2009). A new parareptile with temporal fenestration from the Middle Permian of South Africa. Can. J. Earth Sci. 46, 9-20. doi: 10.1139/E09-001

Müller, J., and Tsuji, L. A. (2007). Impedance-matching hearing in Paleozoic reptiles: evidence of advanced sensory perception at an early stage of amniote evolution. PLOS ONE 2:e889. doi: 10.1371/journal.pone.0000889

Nuñez Demarco, P., Meneghel, M., Laurin, M., and Piñeiro, G. (2018). Was Mesosaurus a fully aquatic reptile? Front. Ecol. Evol. 6:109. doi: $10.3389 /$ fevo.2018.00109

Piñeiro, G., Ferigolo, J., Ramos, A., and Laurin, M. (2012a). Cranial morphology of the Early Permian mesosaurid Mesosaurus tenuidens and the evolution of the lower temporal fenestration reassessed. C. R. Palevol 11, 379-391. doi: 10.1016/j.crpv.2012.02.001

Piñeiro, G., Ramos, A., Goso, C., Scarabino, F., and Laurin, M. (2012b). Unusual environmental conditions preserve a Permian mesosaur-bearing Konservat-Lagerstätte from Uruguay. Acta Palaeont. Pol. 57, 299-318. doi: 10.4202/app.2010.0113

Reisz, R. R., and Scott, D. (2002). Owenetta kitchingorum, sp. nov., a small parareptile (Procolophonia: Owenetidae) from the Lower Triassic of South Africa. J. Vertebr. Paleontol. 22, 244-256. doi: 10.1671/02724634(2002)022[0244:OKSNAS]2.0.CO;2

Rineau, V., Zaragüeta I Bagils, R., and Laurin, M. (2018). Impact of errors on cladistic inference: simulation-based comparison between parsimony and three-taxon analysis. Contr. Zool. 87, 25-40.

Rineau, V., Grand, A., Zaragüeta, R., and Laurin, M. (2015). Experimental systematics: sensitivity of cladistic methods to polarization and character ordering schemes. Contrib. Zool. 84, 129-148.

Ruta, M., and Coates, M. I. (2007). Dates, nodes and character conflict: addressing the lissamphibian origin problem. J. Syst. Palaeontol. 5, 69-122. doi: $10.1017 /$ S1477201906002008
Schoch, R. R., and Sues, H.-D. (2018). Osteology of the Middle Triassic stemturtle Pappochelys rosinae and the early evolution of the turtle skeleton. J. Syst. Palaeontol. 16, 927-965. doi: 10.1080/14772019.2017.1354936

Simmons, M. P. (2012a). Misleading results of likelihood-based phylogenetic analyses in the presence of missing data. Cladistics 28, 208-222. doi: 10.1111/j.1096-0031.2011.00375.x

Simmons, M. P. (2012b). Radical instability and spurious branch support by likelihood when applied to matrices with non-random distributions of missing data. Mol. Phyl. Evol. 62, 472-484. doi: 10.1016/j.ympev.2011.10.017

Simões, T. R., Caldwell, M. W., Palci, A., and Nydam, R. L. (2017). Giant taxon-character matrices: quality of character constructions remains critical regardless of size. Cladistics 33, 198-219. doi: 10.1111/cla.12163

Swofford, D. L. (2003). PAUP* Phylogenetic Analysis Using Parsimony (* and Other Methods). Ver. Version 4.0 build 163. Sinauer Associates.

Tissier, J., Rage, J.-C., and Laurin, M. (2017). Exceptional soft tissues preservation in a mummified frog-eating. Eocene Salamander. PeerJ. 5:e3861. doi: $10.7717 /$ peerj.3861

Tsuji, L. A., Müller, J., and Reisz, R. R. (2010). Microleter mckinzieorum gen. et sp. nov. from the Lower Permian of Oklahoma: the basalmost parareptile from Laurasia. J. Syst. Palaeontol. 8, 245-255. doi: 10.1080/14772010903461099

Tsuji, L. A., Müller, J., and Reisz, R. R. (2012). Anatomy of Emeroleter levis and the phylogeny of the nycteroleter parareptiles. J. Vertebr. Paleontol. 32, 45-67. doi: 10.1080/02724634.2012.626004

von Huene, F. (1941). Osteologie und systematische Stellung von Mesosaurus. Palaeontogr. Abt. A 92, 45-58.

Wegener, A. (1966). The Origin of Continents and Oceans. New York, NY: Dover Publications, Inc.

Conflict of Interest Statement: The authors declare that the research was conducted in the absence of any commercial or financial relationships that could be construed as a potential conflict of interest.

Copyright (c) 2018 Laurin and Piñeiro. This is an open-access article distributed under the terms of the Creative Commons Attribution License (CC BY). The use, distribution or reproduction in other forums is permitted, provided the original author(s) and the copyright owner(s) are credited and that the original publication in this journal is cited, in accordance with accepted academic practice. No use, distribution or reproduction is permitted which does not comply with these terms. 